\title{
NeuroImage
}

ELSEVIER

\section{Lateralization of ventral and dorsal auditory-language pathways in the human brain}

\author{
Geoffrey J.M. Parker, ${ }^{\mathrm{a}, *}$ Simona Luzzi, ${ }^{\mathrm{b}, \mathrm{c}}$ Daniel C. Alexander, ${ }^{\mathrm{d}}$ \\ Claudia A.M. Wheeler-Kingshott, ${ }^{\mathrm{e}}$ Olga Ciccarelli, ${ }^{\mathrm{e}, \mathrm{f}}$ and Matthew A. Lambon Ralph ${ }^{\mathrm{b}}$ \\ a Imaging Science and Biomedical Engineering, University of Manchester, Manchester M13 9PT, UK \\ ${ }^{\mathrm{b}}$ Department of Psychology, University of Manchester, Manchester, UK \\ ${ }^{\mathrm{c}}$ Department of Neuroscience, University of Ancona, Ancona, Italy \\ ${ }^{\mathrm{d}}$ Department of Computer Science, University College London, London, UK \\ ${ }^{\mathrm{e}}$ NMR Research Unit, Institute of Neurology, Queen Square, University College London, London, UK \\ ${ }_{\mathrm{f}}^{\mathrm{f}}$ epartment of Rehabilitation, Brain Injury and Headache, Queen Square, University College London, London, UK
}

Received 24 February 2004; revised 9 August 2004; accepted 30 August 2004

Available online 8 December 2004

Recent electrophysiological investigations of the auditory system in primates along with functional neuroimaging studies of auditory perception in humans have suggested there are two pathways arising from the primary auditory cortex. In the primate brain, a 'ventral' pathway is thought to project anteriorly from the primary auditory cortex to prefrontal areas along the superior temporal gyrus while a separate 'dorsal' route connects these areas posteriorly via the inferior parietal lobe. We use diffusion MRI tractography, a noninvasive technique based on diffusion-weighted MRI, to investigate the possibility of a similar pattern of connectivity in the human brain for the first time. The dorsal pathway from Wernicke's area to Broca's area is shown to include the arcuate fasciculus and connectivity to Brodmann area 40, lateral superior temporal gyrus (LSTG), and lateral middle temporal gyrus. A ventral route between Wernicke's area and Broca's area is demonstrated that connects via the external capsule/uncinate fasciculus and the medial superior temporal gyrus. Ventral connections are also observed in the lateral superior and middle temporal gyri. The connections are stronger in the dominant hemisphere, in agreement with previous studies of functional lateralization of auditory-language processing.

(C) 2004 Elsevier Inc. All rights reserved.

Keywords: Diffusion-weighted imaging; Tractography; Language; Lateralization

* Corresponding author. Imaging Science and Biomedical Engineering, University of Manchester, Oxford Road, Manchester M13 9PT, UK. Fax: +44 (0)161 2755145 .

E-mail address: geoff.parker@man.ac.uk (G.J.M. Parker).

Available online on ScienceDirect (www.sciencedirect.com).

\section{Introduction}

The notion that language is dependent upon both neural centers and the connections between them is well established. In classical aphasiology, Lichtheim's model was comprised of Wernicke's and Broca's areas connected dorsally through the arcuate fasciculus (Lichtheim, 1885). However, modern primate electrophysiological and tracer injection studies suggest two separate pathways connecting the auditory cortex to prefrontal areas (Kaas and Hackett, 1999; Romanski et al., 1999). With analogy to the 'what' and 'where' visual pathways, it has been proposed that, in addition to the dorsal pathway, there is a 'ventral' route, with connections projecting anteriorly along the superior temporal gyrus (Hickok and Poeppel, 2000; Rauschecker, 1998). The two routes may correspond to functional specialization in auditory processing (Rauschecker, 1998; Wise, 2003), with the ventral pathway, like the visual analogue, specialized for recognition of familiar sounds and, in humans, of intelligible speech (Rauschecker, 1998; Scott et al., 2000). There is less agreement about the function(s) of the dorsal stream (Belin and Zatorre, 2000; Romanski et al., 2000), which may be specifically for spatial processing (Rauschecker, 1998; Romanski et al., 1999), or for computing aspects of spectral motion within the acoustic input (Belin and Zatorre, 2000), which may be important for phoneme perception in speech, melody, and timbre in music, as well as mimicry and repetition of speech and other sounds (Belin and Zatorre, 2000; Wise et al., 2001).

Although the neuroanatomy of the monkey auditory network is well established, there is little or no evidence concerning these pathways in the human brain. Unlike some aspects of primate brain function, conclusions regarding auditory-language processing in the human brain drawn from parallels with nonhuman primates must be viewed with caution (Gloor et al., 1997). The purpose of this study was, therefore, to use state-of-the-art magnetic resonance imaging tractography, a noninvasive method for determining 
anatomical connectivity in vivo (Conturo et al., 1999), to investigate connections between regions of the human auditorylanguage system. The technique uses diffusion-weighted imaging, which measures the self-diffusion of tissue water, the movement of which is hindered by microscopic tissue structure. These hindrances have consistent orientation in fibrous tissue such as white matter, which leads to orientation-dependent water mobility (anisotropic diffusion). MRI diffusion measurements characterize this anisotropy and provide an estimate of the dominant axis or axes of fiber bundles in each image voxel. The standard approach is to estimate the diffusion tensor (Basser et al., 1994) from multiple measurements in each voxel and to use the principal axis of the tensor as the fiber orientation estimate. Tractography algorithms use this information to map connectivity noninvasively, typically by following the estimated fiber orientation between voxels to generate streamlines (Conturo et al., 1999; Mori et al., 1999), which correspond to estimated fiber trajectories. Such methods have provided previously inaccessible information regarding, for example, thalamic connectivity in normal subjects (Behrens et al., 2003) and connectivity alterations due to pathology, such as tumors (Guye et al., 2003) and stroke (Pierpaoli et al., 2001). However, single tensor streamline methods are limited in regions where fiber bundles cross, as in these areas the single tensor model fits the data poorly. We instead use recently developed methods that can estimate the orientations of crossing fibers (Alexander et al., 2002; Parker and Alexander, 2003; Tuch et al., 2002). We are particularly interested in the region where the superior longitudinal fasciculus/arcuate fasciculus crosses the corona radiata, due to the involvement of the arcuate fasciculus in auditory-language connectivity (Lichtheim, 1885), mediating communication between Wernicke's and Broca's areas. We constrain our analysis to identify pathways that pass through both Wernicke's and Broca's areas, or their homologues in the nondominant hemisphere. This allows the anatomical routes of connection between these two auditory-language centers to be defined while avoiding the extraction of connections that are not specific to our aims (Conturo et al., 1999; Catani et al., 2002).

\section{Methods}

\section{Data acquisition and reconstruction}

Diffusion-weighted brain data were acquired using a GE Signa 1.5-T scanner with a standard quadrature head coil. Eleven righthanded normal volunteers were studied (six male, five female). Sequence parameters were as follows: cardiac gating (TR $=20$; RR $\sim 20 \mathrm{~s}$ ); 60 axial slices; TE $=95 \mathrm{~ms} ; 54$ noncollinear diffusionweighting directions with a $b$-factor of $1156 \mathrm{~s} \mathrm{~mm}^{-2}$; six acquisitions with a $b$-factor approximately $0 \mathrm{~s} \mathrm{~mm}^{-2} ; \delta=34$ $\mathrm{ms} ; \Delta=40 \mathrm{~ms}$; gradient strength $G=22 \mathrm{mTm}^{-1} ; 96 \times 96$ acquisition matrix, interpolated during reconstruction to $128 \times$ 128; $220 \mathrm{~mm}$ field of view, generating $2.30 \times 2.30 \times 2.30 \mathrm{~mm}^{3}$ voxels as acquired, reconstructed to $1.72 \times 1.72 \times 2.30 \mathrm{~mm}^{3}$ (Jones et al., 1999; Wheeler-Kingshott et al., 2002). The acquisition time for each data set was approximately $20 \mathrm{~min}$, depending on heart rate. Eddy current-induced image distortions in the diffusion-sensitized images were removed using affine multiscale two-dimensional registration (Symms et al., 1997). The volume occupied by the brain was extracted on the $b=0$ images to provide a brain mask using the brain extraction tool (BET) available in the FSL software package (http://www.fmrib.ox. ac.uk). All subjects were scanned with the approval of the joint National Hospital and Institute of Neurology ethics committee and gave written informed consent.

\section{Voxel classification}

We applied the algorithm of Alexander et al. (2002) to identify voxels in which the single tensor model is poor. In these cases, we fit a mixture of two Gaussian densities; otherwise, we use the single tensor model. The principal diffusion directions of the two diffusion tensors in the mixture model provide estimates of the orientations of the crossing fibers.

We define

$d(\hat{\mathbf{k}})=b^{-1}(\log S(\hat{\mathbf{k}}, b)-\log S(\hat{\mathbf{k}}, 0))$,

where $S(\mathbf{k}, b)$ is the signal measurement with diffusion weighting gradient direction $\mathbf{k}$ and diffusion weighting factor $b$. For the single tensor model

$d(\hat{\mathbf{k}})=\hat{\mathbf{k}}^{T} \mathbf{D} \hat{\mathbf{k}}$.

This single tensor is a good model of diffusion in tissues where a single fiber bundle direction is present in an image voxel. In voxels containing $n$ different fiber directions, a multi-Gaussian model is more appropriate (Alexander et al., 2001, 2002; Frank, 2002; Tuch et al., 2002):

$S(\hat{\mathbf{k}}, b)=S(0) \sum_{i=1}^{n} a_{i} \exp \left[-b \hat{\mathbf{k}}^{T} \mathbf{D}_{i} \hat{\mathbf{k}}\right]$

We use a spherical harmonic (SH) model of $d(\mathbf{k})$ to identify voxels in which the single tensor model is poor (Alexander et al., 2002). Thus

$d(\hat{\mathbf{k}})=\sum_{l=0}^{\infty} \sum_{m=-l}^{l} c_{l m} Y_{l m}(\hat{\mathbf{k}})$,

where $Y_{l m}$ is the spherical harmonic of order $l$ and index $m$. Since $d(\hat{\mathbf{k}})$ is real and $d(\hat{\mathbf{k}})=d(-\hat{\mathbf{k}}), c_{l m}=0$ when $l$ is odd and $c_{l m}=(-1)^{m} c_{l-m}$. We truncate the series at order 0,2 , or 4 using the analysis of variance test for deletion of variables to select the best model in each voxel. The $F$ statistic thresholds used for model truncation (see Alexander et al., 2002) are the same for all individuals in the study under the assumption of similar noise levels in all data sets. Truncation at order 0 indicates isotropic diffusion. Truncation at order 2 indicates that the single tensor model is a good approximation so that Eqs. (2) and (4) are equivalent. When fourth-order terms are included in the series, the single tensor fit is poor and we fit the multi-Gaussian model with $n=2$. We assume that we cannot resolve the directions of more than two fibers with the number (54) of diffusion-weighted measurements acquired. To fit the multi-Gaussian model, we use a Levenberg-Marquardt algorithm on data resampled from the spherical harmonic model of $d(\hat{\mathbf{k}})$.

\section{Spatial normalization}

Data were transformed into standard space using the linear registration tool (FLIRT) available in the FSL software package 
(http://www.fmrib.ox.ac.uk). The EPI template available with the SPM package (http://www.fil.ion.ucl.ac.uk) was used to normalize the high-resolution EPI image. The resultant transformation was applied to all fiber tracking output to put all results into standard space.

\section{Volume of interest definition}

The locations and extents of Broca's and Wernicke's areas (which we defined as being Brodmann areas 44 and 22, respectively; Dejerine et al., 1914) were identified in each subject by a single neurologist on the spatially normalized highresolution EPI images in the left hemisphere with the aid of literature sources (Duvernoy et al., 1999; Pfeifer et al., 1936; Talairach and Tournoux, 1988). Wernicke's area and the region of ventrolateral prefrontal cortex homologous to Broca's area in the right hemisphere were identified visually using anatomical landmarks to produce regions analogous to those defined in the left hemisphere.

In both hemispheres, the locations of Broca's area volumes of interest (VOIs) were bordered caudally by the inferior precentral sulcus, dorsally by the inferior frontal sulcus, and extended rostrally to include the opercular region of the inferior frontal gyrus as far as the vertical ramus. Wernicke's area VOIs corresponded approximately to the posterior third of the superior temporal gyrus, bordered caudally and dorsally by the angular and supramarginal gyri, respectively. We defined the rostral border of Wernicke's area as the position of Heschl's sulcus.

Volumes of interest (VOIs) were traced across the twodimensional slices identified as containing the desired area using the DispImage package (Plummer, 1992). VOIs were defined to encompass the grey matter relating to each area and neighboring gyral white matter to allow white matter fiber tracking. The VOIs were converted into mask images and transformed back into native space for fiber tracking. The results of the tracking process were transformed once more into standard space to allow group comparisons and localization of connectivity volumes with reference to a standard atlas (Talairach and Tournoux, 1988).

\section{Path tracking}

Tracking analysis was performed using a dedicated software package developed for the purpose of visualization and navigation through the diffusion data volume using a multiplanar view. A modified streamline method is used to propagate paths through the multitensor field. A step in the streamline propagation process is defined:

$\mathbf{X}(l+1)=\mathbf{X}(l)+\mathbf{w}(l) \delta t$,

where $\mathbf{X}$ is the position in $\mathfrak{R}^{3}$ of the streamline at point $l$ on its length, $\mathbf{w}(l)$ is the propagation direction at this point, and $\delta t$ is the step size. With a single tensor model of diffusion, we use trilinear interpolation of surrounding tensor elements to obtain an interpolated tensor at nongrid points of the image. We compute the principal eigenvector of the interpolated tensor to give $\mathbf{w}(l)$. In the case where one or more of the image voxels involved in the interpolation is described by more than one tensor, we employ selection rules to ensure the most appropriate tensor is included in the interpolation. If $\mathbf{D}_{i}(\mathbf{p})$ is one of $n$ tensors present at an image location p contributing to the interpolation, then we select the tensor with index

$\max _{i}\left(\left|\Gamma\left(\mathbf{D}_{i}(\mathbf{p})\right) \cdot \mathbf{w}(l-1)\right|\right)$,

where $\boldsymbol{\Gamma}\left(\mathbf{D}_{i}(\mathbf{p})\right)$ is the principal eigenvector of the $i$ th tensor at p. This formulation ensures that when fiber crossing is detected, the tensor representing the fiber with an orientation closest to that of the current streamline propagation direction is chosen to influence further propagation. Stopping criteria for the streamlines were set so that tracking terminated if a pathway extended into grey matter (order 0 voxel classification). No penalty was imposed on pathway curvature. Step size was set to $0.23 \mathrm{~mm}$.

Streamline propagation was constrained using pairs of VOIs. The VOI information was used based on the procedure of Conturo et al. (1999). Tracking was started from the center of every voxel in the brain that was of order 2 or greater and a pathway was accepted if and only if it passed through both regions (i.e., Broca's area and Wernicke's area in the left hemisphere experiments; the homologous areas in the right hemisphere experiments). The voxels that were encountered by an accepted pathway were identified along its route, and the number of times each voxel was encountered throughout the whole experiment was recorded. This generated maps of voxels that were connected to the two areas of interest. The analysis for two subjects failed to show any connections between the VOIs. This is likely to be caused by a failure in the voxel classification stage of the data processing due to a poor signal to noise ratio in these cases. Modest improvements in data quality are likely to ameliorate these problems in future studies.

\section{Variability maps}

Variability group maps were constructed using binary masks of the connected volumes in standard (normalized) space and of the VOI volumes in standard space from each individual. The masks were averaged, thus indicating the degree of spatial variability and overlap of the identified connections or VOIs between subjects (Ciccarelli et al., 2003). A voxel commonality value $\mathrm{C}$ of 1.0 indicates that each individual had a connection or VOI identified in this voxel; $C=0.0$ indicates that none of them did.

\section{Lateralization index}

To assess lateralization of the connected volume between hemispheres, the lateralization index LI was calculated:

$$
\mathrm{LI}(C)=\frac{N(C)_{\text {left }}-N(C)_{\text {right }}}{0.5 \times\left(N(C)_{\text {left }}+N(C)_{\text {right }}\right)},
$$

where $N(C)_{\text {left }}$ and $N(C)_{\text {right }}$ are the total number of voxels in standard space in the connected volumes above a threshold value in $C$ of the left and right hemispheres, respectively. This was calculated at a range of thresholds in $C$ to allow us to determine whether lateralization was caused by more or less variable connected volume within the group. Lateralization was also determined for the volumes occupied by the VOIs used for tracking. 
Connecting volume

The connecting volume $V(C)$ is the volume in standard space occupied by voxels above the commonality threshold $C$.

\section{Results}

\section{Volume of interest lateralization}

Fig. 1 shows the population-averaged commonality map for VOIs defined in the left and right hemispheres. The VOIs encompass both white and grey matter in the vicinity of Broca's area in the ventrolateral prefrontal region and Wernicke's area in the posterior superior temporal gyrus. The right hemisphere homologues are similarly located. The volumes of the VOIs defined for both Broca's and Wernicke's areas are significantly larger in the left hemisphere. For Broca's area and its right hemisphere homologue, the mean volumes were $5519 \pm 2517$ $\mathrm{mm}^{3}$ (median $4848 \mathrm{~mm}^{3}$ ) and $2943 \pm 563 \mathrm{~mm}^{3}$ (median 2904 $\left.\mathrm{mm}^{3}\right)$, respectively $(P=0.007$, paired $t$ test; LI $=0.55 \pm 0.29$ (median 0.54)); for Wernicke's area the mean volumes were $6582 \pm 808 \mathrm{~mm}^{3}\left(\right.$ median $\left.6408 \mathrm{~mm}^{3}\right)$ in the left hemisphere and $4388 \pm 702 \mathrm{~mm}^{3}$ (median $4344 \mathrm{~mm}^{3}$ ) in the right hemisphere $(P<0.001$, paired $t$ test; LI $=0.40 \pm 0.21$ (median 0.49$)$ ). The variability in the volumes occupied by the regions in the left hemisphere is greater than in the right, but much of this may be accounted for by the presence of a single outlier in the identified Broca's area volume. Fig. 2 shows the volumes recorded for the VOIs defined in each of the 11 subjects.

\section{Connection lateralization}

Fig. 3 shows the result of constrained multifiber streamline tracking between the VOIs defined in a single individual. The dorsal pathway involving the arcuate fasciculus plus a connection to Brodmann area 40 (supramarginal gyrus) appears in both hemispheres. A ventral connection to the superior temporal gyrus is also evident. This ventral route is only apparent in the left hemisphere. Overall, more connecting voxels are identified in the left than the right hemisphere.

\section{Left hemisphere variability}

Fig. 4 shows the group variability map for the volume of connection in the left hemisphere. Consistent connection is observed between Broca's area and Wernicke's area via the arcuate fasciculus, with all 9 (of 11) successfully traced brains indicating this fiber bundle. The connection involving Brodmann area 40 also appears across all successfully traced brains, although the lateral extent of this connection varied. There was less consistent connectivity in the inferior direction including connection to the

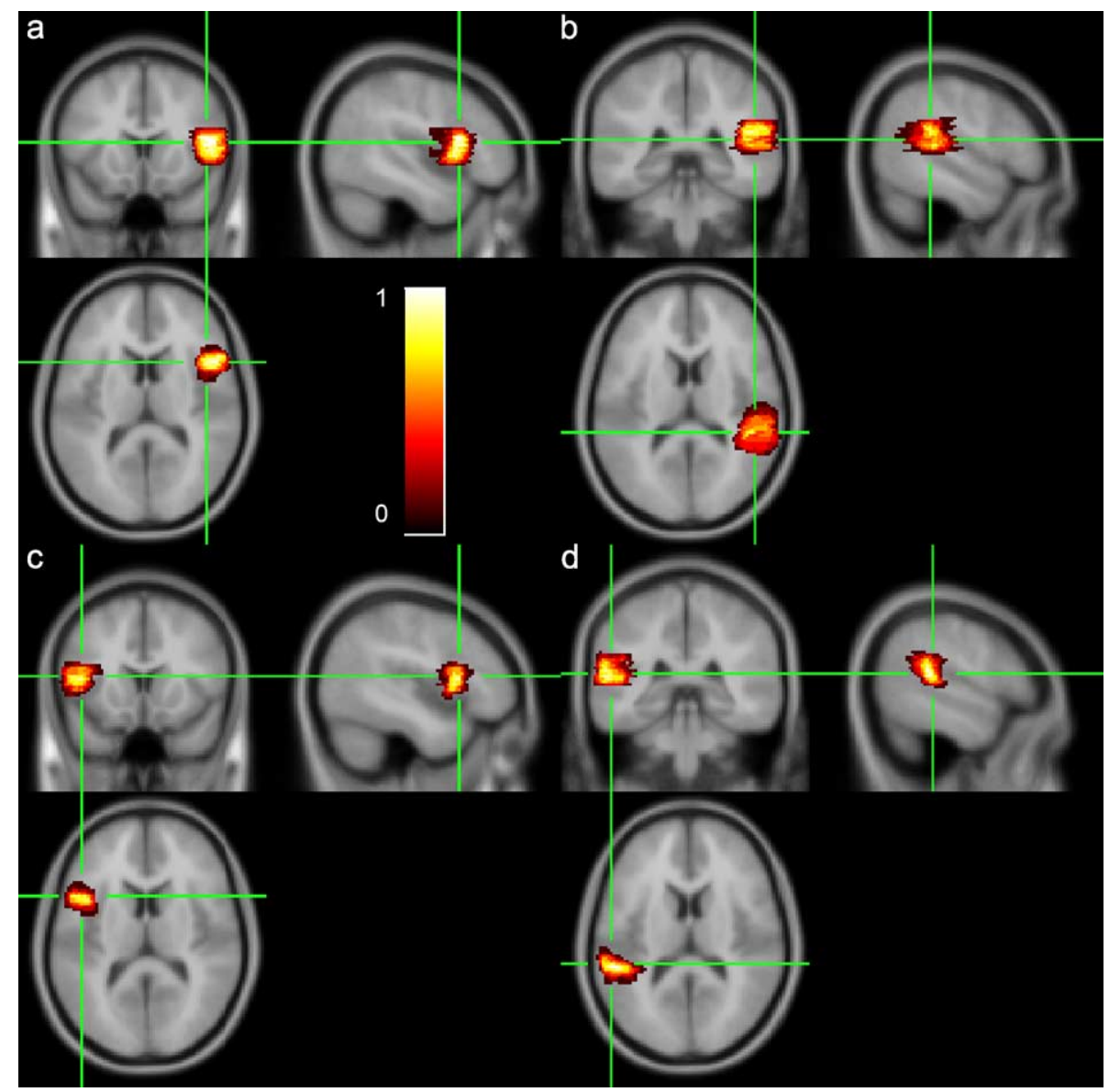

Fig. 1. Group variability maps showing VOIs used to define Broca's area (a), Wernicke's area (b), and right hemisphere homologues (c and d) as used to constrain the tracking process. Radiological viewing convention used. Color bar expresses degree of commonality $C$. 


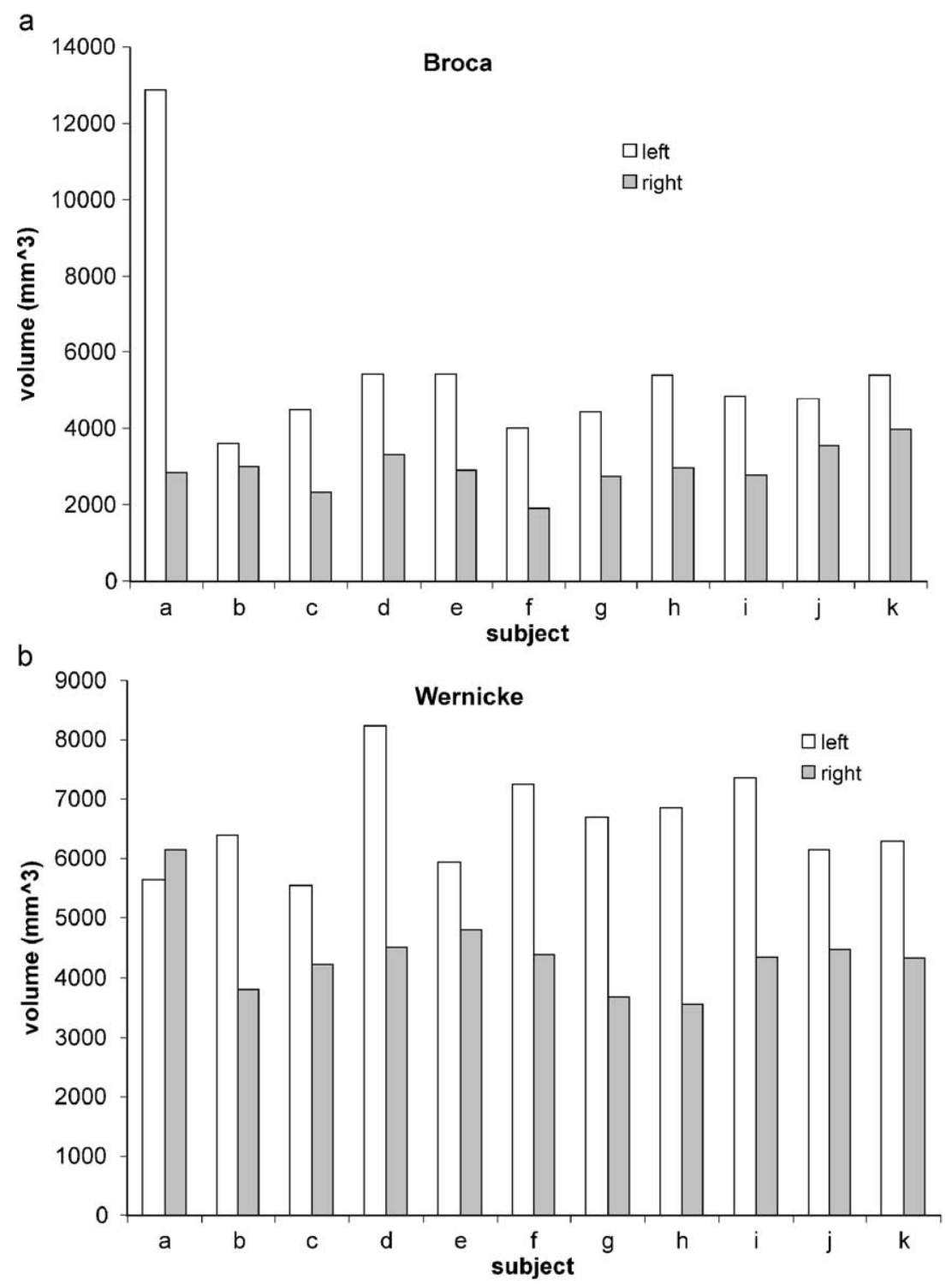

Fig. 2. Volumes occupied by VOIs in left and right hemispheres. (a) Broca's area and right hemisphere homologue; (b) Wernicke's area in left and right hemisphere.

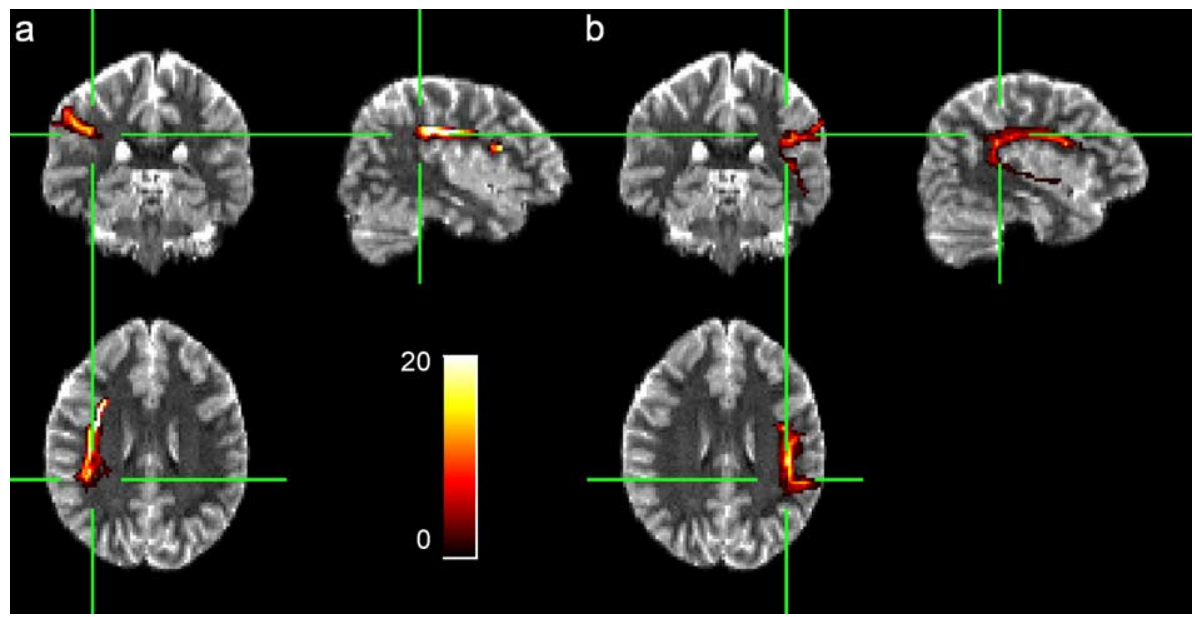

Fig. 3. Single individual showing differences in connecting volume between right (a) and left (b) hemispheres (radiological viewing convention used). Overlay onto high-resolution EPI volume. Color bar expresses number of streamline paths identified passing through each voxel. 


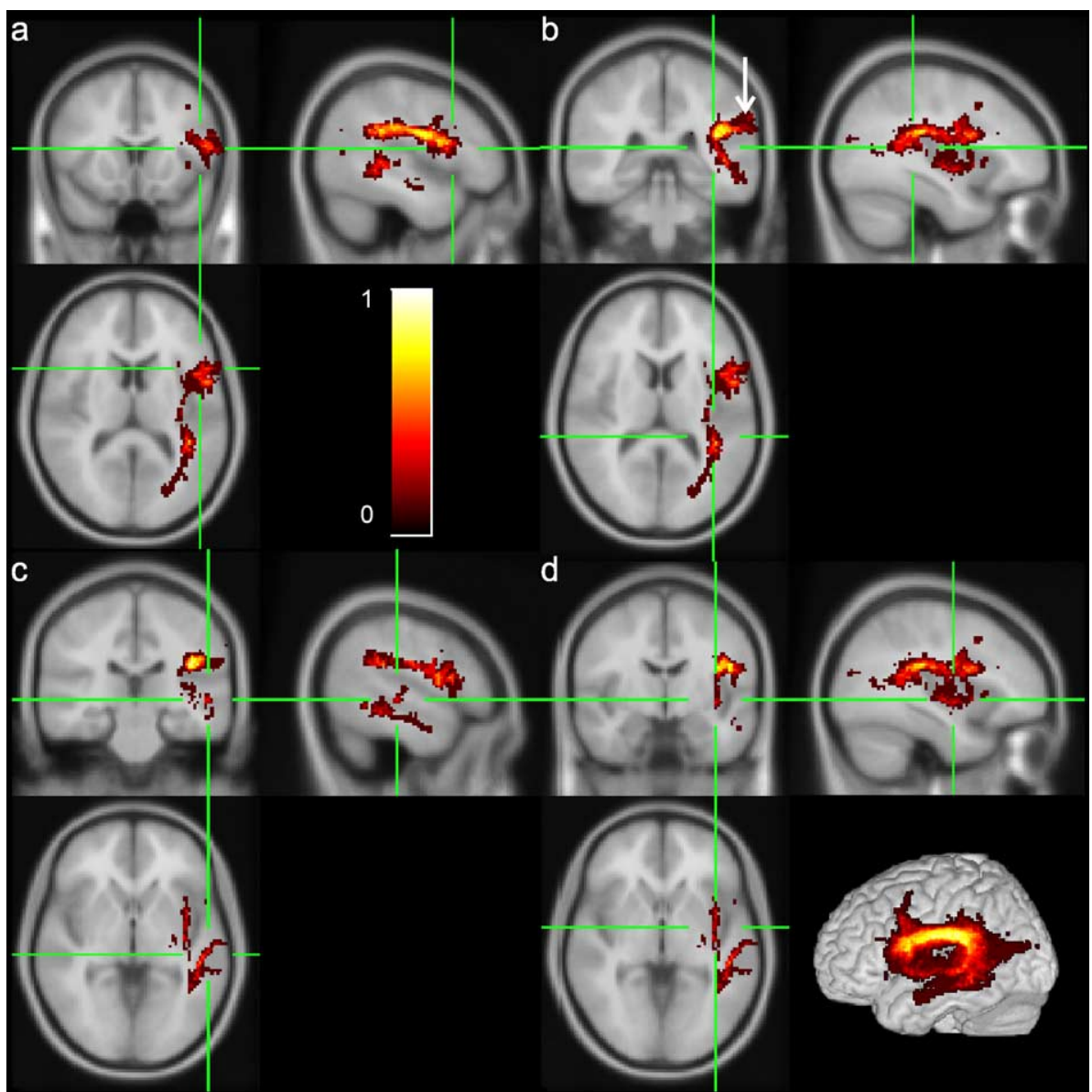

Fig. 4. Group variability map in the left hemisphere. Crosshairs at stated Talairach coordinates: (a) Broca's area $(-45,15,10)$, (b) Wernicke's area $(-35,-35,10)$. Also note consistent connection to Area 40 (arrow). (c) Connection to superior temporal gyrus. Also note evidence of connection to middle temporal gyrus and via external capsule/uncinate fasciculus. (d) Connection via external capsule/uncinate fasciculus between Broca's area and Wernicke's area. Brain surface rendering with embedded spatial distribution of commonality map also shown. Color bar expresses degree of commonality $C$.

lateral superior and middle temporal gyri, plus connection via the medial superior temporal gyrus, forming a ventral route between the two constraining VOIs. Four of the 11 subjects $(45 \%$ of successfully traced brains) exhibit this ventral connection between Broca's and Wernicke's area, which passes either via the uncinate fasciculus or via the slightly superior part of the external capsule. The overall observed pattern of connection is summarized schematically in Fig. 5.

\section{Right hemisphere variability}

The group variability map for the right hemisphere connection volume is shown in Fig. 6 and is summarized schematically in Fig. 7. A smaller volume of connection is observed overall, though the consistent connection via the arcuate fasciculus is comparable to that observed in the left hemisphere. Likewise, the connection to Brodmann area 40 is also consistently present. In contrast, the connections to the superior and middle temporal gyri and via the external capsule/uncinate fasciculus are lacking in the right hemisphere.

Fig. 8 shows a plot of left and right hemisphere volumes of connection and lateralization index LI as a function of commonality value $C$. The left hemisphere has a larger connected volume at all values of $C$ and, therefore, $\mathrm{LI}$ is positive for all values of $C$.

\section{Assessment of VOI volume bias}

A possible cause for the observed connecting volume lateralization is the difference in VOI volume between the hemispheres

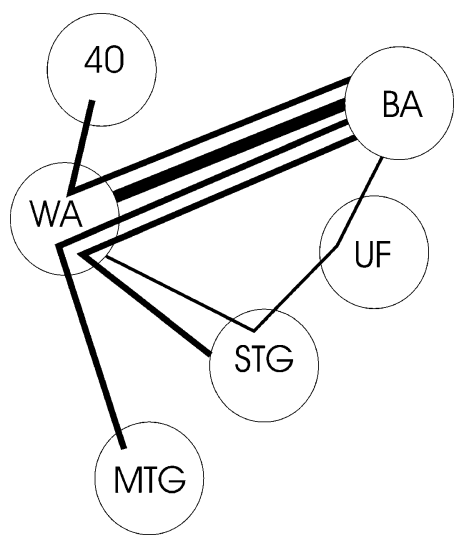

Fig. 5. Schematic of connections observed across the group in the left hemisphere (Fig. 4). Weight of connecting lines approximates commonality value $C . \mathrm{BA}=$ Broca's area; $\mathrm{WA}=$ Wernicke's area; $\mathrm{UF}=$ uncinate fasciculus/external capsule; $\mathrm{STG}=$ superior temporal gyrus; $\mathrm{MTG}=$ middle temporal gyrus. 


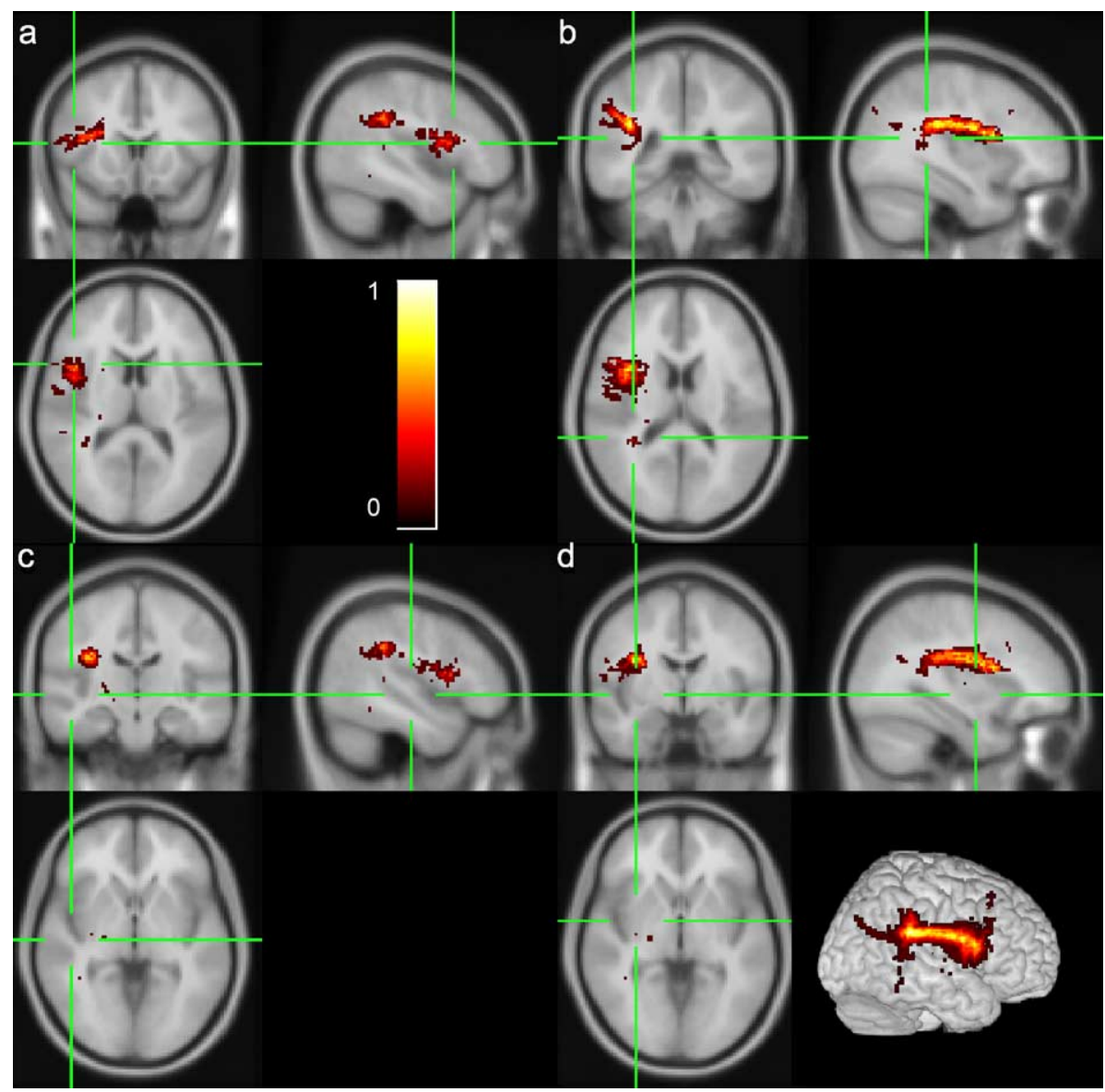

Fig. 6. Group variability map in the right hemisphere. Crosshairs at stated Talairach coordinates: (a) analogue of Broca's area $(45,15,10)$, (b) analogue of Wernicke's area $(35,-35,15)$. Also note consistent connection to Area 40 (b), lack of connection to temporal lobe (c), and lack of connection via external capsule/uncinate fasciculus (d). Brain surface rendering with embedded spatial distribution of commonality map also shown. Color bar expresses degree of commonality $C$.

identified above. While cortical volume lateralization may be expected and has been reported many times previously (see Discussion), it is nevertheless possible that the difference in VOI volumes between the hemispheres may be leading to an artifactual

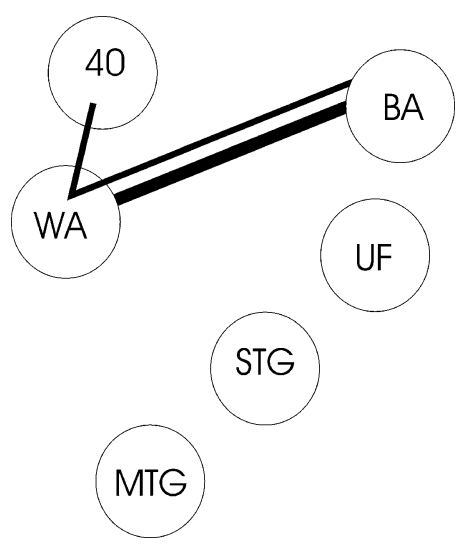

Fig. 7. Schematic of connections observed across the group in the right hemisphere. Weight of connecting lines approximates commonality value $C$. $\mathrm{BA}=$ Broca's area; $\mathrm{WA}=$ Wernicke's area; $\mathrm{UF}=$ uncinate fasciculus/ external capsule; $\mathrm{STG}=$ superior temporal gyrus; $\mathrm{MTG}=$ middle temporal gyrus. Many connections that are present in the left hemisphere (Fig. 5) are lacking. lateralization in connecting fiber tract volumes. To assess the contribution that VOI volumes make to our connecting volume measurements, we plotted connecting volume as a function of the combined volume of the Broca's area and Wernicke's area VOIs (Fig. 9). Using linear regression of connecting volume against combined VOI volume, for the left hemisphere $r^{2}=0.092$, for the right hemisphere $r^{2}=0.030$. A test against the null hypothesis of no relationship between VOI volumes and connecting volumes indicates that the null hypothesis holds for both hemispheres ( $P=$

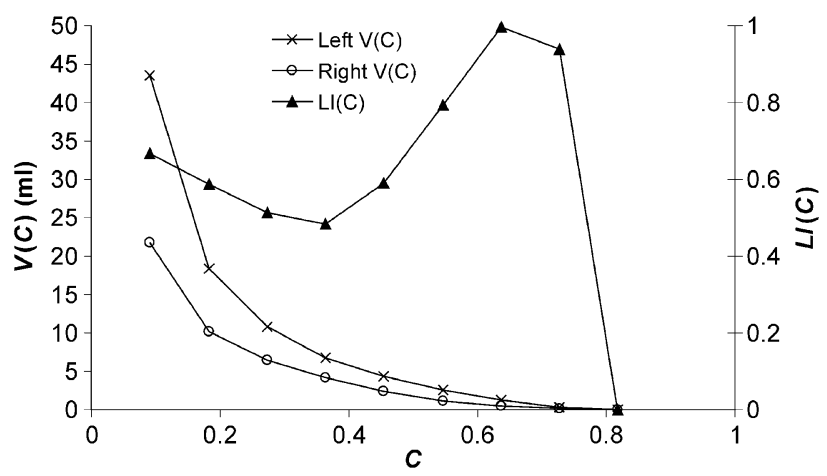

Fig. 8. Connecting volume $V(C)$ and lateralization index $\operatorname{LI}(C)$ as a function of commonality value $C$. 


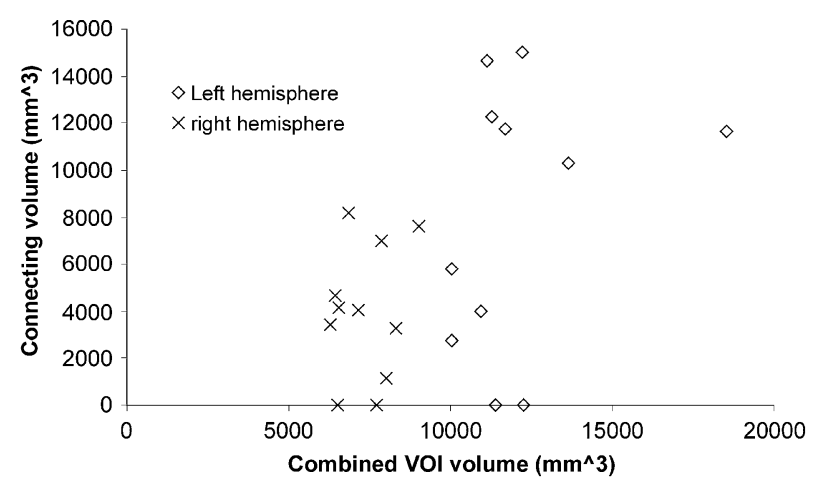

Fig. 9. Plot of connecting volume versus VOI volume for each volunteer. Note the lack of relationship between the combined (Broca's area plus Wernicke's area) VOI volumes within each hemisphere and the connecting volumes. Also note the clear difference in VOI volume between the hemispheres, but the presence of some overlap in connecting volume.

0.365 and $P=0.609$ for the left and right hemispheres, respectively). There is therefore no evidence of a relationship between connecting volume and VOI volume in either hemisphere. Fig. 9 also reiterates the clear separation in VOI volume between the hemispheres, while indicating some overlap in connecting volumes (although the difference is statistically significant ( $P=$ 0.019 , paired $t$ test, two-tailed)).

\section{Discussion}

As one would expect from classical aphasiology, the in vivo tractography analysis of the human brain identified a major connection between Wernicke's and Broca's area via the arcuate fasciculus. This was consistently apparent in both hemispheres. Our analysis also found evidence for a second, ventral pathway between these auditory-language centers, the existence of which has been suggested as a result of nonhuman primate studies (Rauschecker, 1998; Hickok and Poeppel, 2000). The volume occupied by the identified connective pathways in the left hemisphere was larger than in the right, implying more extensive anatomical connectivity, as has previously been hypothesized (Hickok and Poeppel, 2000). The second, ventral route was only identified in the left hemisphere, which is consistent with recent functional neuroimaging findings that show activation in only the left hemisphere activation for processing intelligible speech (Romanski et al., 1999; Scott et al., 2000).

Postmortem studies have previously demonstrated differences in white matter tract volumes between dominant and nondominant hemispheres. Bürgel et al. (1999) showed larger optic radiation volumes in the left hemisphere (right-handed population) and Rademacher et al. (2001) demonstrated similar lateralization of the pyramidal tracts. These studies give us confidence that tractspecific functional dominance-related volume asymmetry is plausible. However, tract lateralization in the auditory system cannot be presupposed, as it has been shown to be absent in a postmortem study of the acoustic radiation in 10 brains (Rademacher et al., 2002). However, an imaging study of 100 righthanded volunteers found clear evidence for white matter volume left-sided lateralization in lateral frontal and temporal lobes (Pujol et al., 2002). We are unaware of any human studies (postmortem or imaging) that have attempted to characterize the specific pathways we have studied here, but, given the wealth of anatomical and functional evidence for auditory-language network lateralization, our findings appear credible.

Our manual delineation of VOIs showed strong evidence of lateralization of inferior frontal gyrus (containing Broca's area in the left hemisphere) and Wernicke's area volumes, consistent with previous findings (see for example Amunts et al., 1999; Good et al., 2001; Pujol et al., 2002; Shapleske et al., 1999; Toga and Thompson, 2003, and references therein). The additional variability in VOI size we observe in the left hemisphere is also consistent with previous findings that link cortical areas showing greatest asymmetry with those showing greatest gyral pattern variability across subjects (Toga and Thompson, 2003).

The finding of connection to the lateral superior temporal gyrus (LSTG) (Fig. 4) in the left hemisphere only $(P<0.001)$ is consistent with findings from functional imaging studies that have identified this area as being involved with the comprehension of intelligible speech (Scott, 2000). Our interpretation of these findings is that this region is connected to the Wernicke-Broca axis via the posterior temporal lobe dorsal route, which is consistent with previous fiber tracking studies (Catani et al., 2002), and not via an anterior temporal lobe ventral route. While the design of our experiment does not preclude a direct connection to Broca's area alone via an anterior route (Romanski et al., 1999; Wise, 2003), it does mean that there is no evidence to support a ventral route encompassing all three regions. This is perhaps not surprising, as interconnections between language-related areas along the superior temporal gyrus (i.e., for our experiment Wernicke's area and more anterior LSTG) are likely to be short-range and intracortical, rather than involving white matter fiber bundles (Wise, 2003); current diffusion-weighted MRI acquisition methods and analysis are not well suited to extracting intracortical connections in vivo. Our results do, however, raise the possibility of interconnection between LSTG, Wernicke's area, and Broca's area via the dorsal, arcuate fasciculus route in the left hemisphere only, which is consistent with the lateralization of function for intelligible speech and phonetic cues to this region (Scott et al., 2000).

The results of primate electrophysiological and invasive tract tracing studies have led to the suggestion that a ventral language pathway may pass via the uncinate fasciculus, linking the anterior temporal lobe to higher cognitive processing areas in the frontal lobe (Romanski et al., 1999). Due to the relatively coarse resolution of the EPI data used in diffusion-weighted imaging and the level of precision associated with the spatial normalization process, it is difficult to state with confidence whether the route of connection we observe does indeed pass through the uncinate fasciculus or more superiorly in the anterior external capsule. However, it is clear that the identified pathway passes in a distinct anterior-posterior orientation, which is at odds with the classical picture of the uncinate fasciculus as a hook-like structure connecting the extreme anterior portion of the temporal lobe to the frontal lobe. Additionally, as discussed above, the observed pathway does not appear to connect the anterior LSTG with Broca's area, as would be expected if it represented the same pathway hypothesized based on animal data. It seems likely, therefore, that the ventral Wernicke-Broca connection that we observe is a distinct medial pathway connecting the posterior superior temporal gyrus with ventrolateral prefrontal areas, without involvement of the anterior LSTG areas.

The tractography analysis also identified a pathway linking to Brodmann area 40 (the supramarginal gyrus) bilaterally. Functional 
imaging studies have implicated this area in a number of languagerelated tasks including overt articulation (Wise et al., 2001), syllable discrimination and identification, and other tasks that require explicit attention to segmental information (Hickok and Poeppel, 2000). Like other parietal areas, this language function may reflect a more general interface between perceptual and motor systems, which have been mirrored in recent neural network models of phonological processes (Plaut and Kello, 1999).

As we were only able to identify left hemisphere ventral connections in a minority of the individuals studied, we conclude that our detection of these pathways is at the limits of the sensitivity of the fiber tracking technique we have employed. We speculate that the observed pattern of left hemisphere connection may also exist in the remaining individuals, but at a level below our sensitivity threshold. Similarly, we cannot discount the possibility of a similar right hemisphere ventral connection pattern below our sensitivity threshold. However, it seems likely that any such right hemisphere white matter connecting tissue occupies a smaller volume than the analogous network in the left hemisphere or is entirely lacking. The sensitivity of the tracking method that we have used is likely to be determined by a combination of factors, including noise levels, the $b$ value, the model-fitting accuracy to the observed diffusion signal, and both the spatial and angular resolution of our data acquisition. Improvements to these factors, or more sensitive tracking methods (for example probabilistic methods; Behrens et al., 2003; Parker and Alexander, 2003; Parker et al., 2003) may in the future clarify our observations. Probabilistic techniques identify the same possible route of connection as deterministic methods, such as that used here, but additionally provide a measure of connection confidence. In addition to the 'deterministic' connection, they also provide a confidence in connection to other regions. 'False negative' connections from a deterministic tracking measurement often appear as connections with a nonzero confidence when using a probabilistic measurement, thus providing higher sensitivity.

The simple multiple-fiber reconstruction we use here has several limitations. Tuch et al. (2003) recently showed evidence that more than two fiber populations can exist and be resolved within a single voxel. Here we reconstruct a maximum of two directions per voxel. The data acquisition of Tuch et al. (2003) is significantly different to that used in our work. Our measurements were performed with far fewer orientational samplings, with a finer spatial resolution, and with a greater imaging coverage. Simulations with mixtures of three Gaussian densities show that voxel classification does not reliably distinguish three-fiber crossings from isotropic diffusion with our acquisition scheme. Furthermore, the method resolves two fiber directions only when the fibers have similar volume fraction and the directions are well separated. The angular resolution with which we are able to distinguish distinct populations is limited by the data sampling scheme and data noise levels. The model selection process classifies crossings of low angular separation as single tensor voxels. Despite these limitations, our ability to distinguish more than a single fiber population in many areas of the brain means that we are able to utilize far more information than is available with 'classical' diffusion tensor imaging. This means that the fiber tracking process is less likely to be prone to systematic orientational errors (albeit with a likely higher level of random noise). Multiple-fiber reconstruction methods and their use in tractography are active areas of research. More sophisticated techniques will undoubtedly expand the capabilities of fiber tracking methods in the future.
Two data sets in the study were unusable due to a failure in voxel classification. An assumption was made that all data sets had approximately equivalent noise levels, allowing the $F$ thresholds used for determination of model order to be kept constant across all volunteers, and allowing calibration for $F$ thresholds for the experimental setup. Although this appears reasonable as all data were acquired on the same scanner with the same protocol, in two subjects it appears that this assumption was flawed. These two data sets were not included in the analysis.

We used anatomical landmarks to identify the volumes of interest required for the constrained tracking process. As, due to the inherent lateralization (see above for discussion of previous reports of anatomical lateralization), it was not possible to blind for hemisphere during the generation of these VOIs, it is possible that bias may be introduced into the left/right volume definition. However, the use of well-defined anatomical boundaries to the regions that we are interested in provides a limitation on the scope for bias beyond genuine anatomical variability. Additionally, we have shown that there is no intrahemisphere correlation between the VOI volumes we defined and the resulting connecting volumes (Fig. 9), implying that even if bias was present it is unlikely to have had a significant impact. However, as all VOIs were defined by a single individual, we cannot comment on any inter-rater bias, which represents a limitation of our study. An alternative approach to VOI definition would be to use functional imaging data to identify their position. However, even if functional information was used to define Broca's and Wernicke's areas, the design of an experiment with which to identify the functional location of the right hemisphere homologue of Broca's area would pose serious challenges due to the inherent lateralization of language function. Automated gyral identification techniques may in the future provide a more objective method for anatomical identification of these structures (Toga and Thompson, 2003), but at the current time, expert manual definition is the only practical option available. Even if such an automated approach was used, there is still a requirement to identify involved white matter within which to initiate fiber tracking, as it is not currently possible to trace fiber tracts into gyral grey matter with confidence. Our solution to this problem was to include gyral white matter in our VOIs, under the assumption that this white matter is highly likely to connect the overlying functional region.

Fig. 4 shows evidence of some occipital connectivity to both defined VOIs (this was found in two individuals in the left hemisphere only). It is possible that in this case we are observing part of the inferior fronto-occipital fasciculus, which is known to connect the ventrolateral frontal lobes and pass via the external capsule (Catani et al., 2002), consistent with our findings. It is possible that the medial ventral route we have identified as connecting Wernicke's area and Broca's area is in fact at least a portion of the inferior fronto-occipital fasciculus. If this is indeed the case, it is difficult to explain why the identified ventral pathway is identified only in the left hemisphere, unless the inferior frontooccipital fasciculus also shows significant structural lateralization. Further investigations into the parcellation of this fiber bundle should in the future clarify this point.

Although it is possible to infer human neuroanatomical networks from animal models, diffusion MRI tractography provides a method to measure patterns of connection within the human brain in vivo. This is especially important for areas related to higher cognitive function and language that may not be found in nonhuman species, and may be mirrored by significant differences 
in human neuroanatomy (Gloor et al., 1997). This study uses tractography to explore the network of connections relating to language in the normal brain. Future studies will use the same technique to quantify the status of the network immediately after brain damage in aphasic patients and to measure changes in this network that may underpin the types of language recovery that functional neuroimaging is now beginning to identify (Blank et al., 2003; Leff et al., 2002).

\section{Acknowledgments}

This work was supported in part by the Medical Research Council and the Engineering and Physical Sciences Research Council of Great Britain Interdisciplinary Research Collaboration From Medical Images and Signals to Clinical Information under Grant GR/N14248/01. We would also like to thank the Wellcome Trust (SL), the MS Society of Great Britain and Northern Ireland (CAMW-K), and Teva Pharmaceuticals (OC) for providing support for individuals involved in this work. We are particularly grateful to Prof. Alan Thompson for his support for the early phase of this study. Color overlays generated using the MRIcro package (www.mricro.com).

\section{References}

Alexander, A.L., Hasan, K.M., Lazar, M., Tsuruda, J.S., Parker, D.L., 2001. Analysis of partial volume effects in diffusion-tensor MRI. Magn. Reson. Med. 45, 770-780.

Alexander, D.C., Barker, G.J., Arridge, S.R., 2002. Detection and modelling of non-Gaussian apparent diffusion coefficient profiles in human brain data. Magn. Reson. Med. 48, 331-340.

Amunts, K., Schleicher, A., Bürgel, U., Mohlberg, H., Uylings, H.B.M., Zilles, K., 1999. Broca's region revisited: cytoarchitecture and intersubject variability. J. Comp. Neurol. 412, 319-341.

Basser, P.J., Mattiello, J., Le Bihan, D., 1994. MR diffusion tensor spectroscopy and imaging. Biophys. J. 66, 259-267.

Behrens, T.E.J., Johansen-Berg, H., Woolrich, M.W., Smith, S.M., WheelerKingshott, C.A.M., Boulby, P.A., Barker, G.J., Sillery, E.L., Sheehan, K., Cicarelli, O., Thompson, A.J., Brady, J.M., Matthews, P.M., 2003. Noninvasive mapping of connections between human thalamus and cortex using diffusion imaging. Nat. Neurosci. 6, 750-757.

Belin, P., Zatorre, R.J., 2000. 'What', 'where' and 'how' in auditory cortex. Nat. Neurosci. 3, 965-966.

Blank, S., Bird, H., Turkheimer, F., Wise, R., 2003. Speech production after stroke: the role of the right pars opercularis. Ann. Neurol. 54, 310-320.

Bürgel, U., Schormann, T., Schleicher, A., Zilles, K., 1999. Mapping of histologically identified long fiber tracts in human cerebral hemispheres to the MRI volume of a reference brain: position and spatial variability of the optic radiation. NeuroImage 10, 489-499.

Catani, M., Howard, R.J., Pajevic, S., Jones, D.K., 2002. Virtual in vivo interactive dissection of white matter fasciculi in the human brain. NeuroImage 17, 77-94.

Ciccarelli, O., Toosy, A.T., Parker, G.J.M., Wheeler-Kingshott, C.A.M., Barker, G.J., Miller, D.H., Thompson, A.J., 2003. Diffusion tractography based group mapping of major white-matter pathways in the human brain. NeuroImage 19, 1545-1555.

Conturo, T.E., Lori, N.F., Cull, T.S., Akbudak, E., Snyder, A.Z., Shimony, J.S., McKinstry, R.C., Burton, H., Raichle, M.E., 1999. Tracking neuronal fiber pathways in the living human brain. Proc. Natl. Acad. Sci. U. S. A. 96, 10422-10427.

Dejerine, J., 1914. Sémiologie des affections du système nerveux. Masson, Paris.
Duvernoy, H.M., 1999. The Human Brain Surface, Blood Supply and Three-Dimensional Sectional Anatomy. Springer, Wein, New York.

Frank, L.R., 2002. Characterization of anisotropy in high angular resolution diffusion-weighted MRI. Magn. Reson. Med. 47, 1083-1099.

Gloor, P., 1997. The Temporal Lobe and The Limbic System. Oxford Univ. Press, Oxford.

Good, C.D., Johnsrude, I.S., Ashburner, J., Henson, R.N., Friston, K.J., Frackowiak, R.S., 2001. A voxel-based morphometric study of ageing in 465 normal adult human brains. NeuroImage 14, 21-36.

Guye, M., Parker, G.J.M., Symms, M., Boulby, P., Wheeler-Kingshott, C.A., Salek-Haddadi, A., Barker, G.J., Duncan, J.S., 2003. Combined functional MRI and tractography to demonstrate the connectivity of the human primary motor cortex in vivo. NeuroImage 19, 1349-1360.

Hickok, G., Poeppel, D., 2000. Towards a functional neuroanatomy of speech perception. Trends Cognit. Sci. 4, 131-138.

Jones, D.K., Horsfield, M.A., Simmons, A., 1999. Optimal strategies for measuring diffusion in anisotropic systems by magnetic resonance imaging. Magn. Reson. Med. 42, 515-525.

Kaas, J.H., Hackett, T.A., 1999. 'What' and 'where' processing in auditory cortex [news]. Nat. Neurosci. 2, 1045-1047.

Leff, A., Crinion, J., Scott, S., Turkheimer, F., Howard, D., Wise, R., 2002. A physiological change in the homotopic cortex following left posterior temporal lobe infarction. Ann. Neurol. 51, 553-558.

Lichtheim, L., 1885. On aphasia. Brain 7, 433-484.

Mori, S., Crain, B.J., Chacko, V.P., van Zijl, P.C.M., 1999. Threedimensional tracking of axonal projections in the brain by magnetic resonance imaging. Ann. Neurol. 45, 265-269.

Parker, G.J.M., Alexander, D.C., 2003. Probabilistic Monte Carlo based mapping of cerebral connections utilising whole-brain crossing fibre information. Lect. Notes Comput. Sci. 2737, 684-695.

Parker, G.J.M., Haroon, H.A., Wheeler-Kingshott, C.A.M., 2003. A framework for a streamline-based probabilistic index of connectivity (PICo) using a structural interpretation of MRI diffusion measurements. J. Magn. Reson. Imag. 18, 242-254.

Pfeifer, R.A., 1936. In: Bumke, O., Foesrter, O. (Eds.), Handbook Der Neruologie. Springer Verlag, Berlin, pp. 523-626.

Pierpaoli, C., Barnett, A., Pajevic, S., Chen, R., Penix, L., Virta, A., Basser, P., 2001. Water diffusion changes in Wallerian degeneration and their dependence on white matter architecture. NeuroImage 13, $1174-1185$.

Plaut, D.C., Kello, C.T., 1999. In: MacWhinney, B. (Ed.), The Emergence of Language. Lawrence Erlbaum Associated, Inc., Mahweh, NJ.

Plummer, D.L., 1992. DispImage: a display and analysis tool for medical images. Rev. Neuroradiol. 5, 489-495.

Pujol, J., Lopez-Sala, A., Deus, J., Cardoner, N., Sebastian-Galles, N., Conesa, G., Capdevilla, A., 2002. The lateral asymmetry of the human brain studied by volumetris magnetic resonance imaging. NeuroImage $17,670-679$.

Rademacher, J., Bürgel, U., Geyer, S., Schormann, T., Schleicher, A., Freund, H.-J., Zilles, K., 2001. Variability and asymmetry in human precentral motor system-A cytoarchitectonic and myeloarchitectonic brain mapping study. Brain 124, 2232-2258.

Rademacher, J., Bürgel, U., Zilles, K., 2002. Stereotaxic localization, intersubject variability, and interhemispheric differences of the human auditory thalamocortical system. NeuroImage 17, 142-160.

Rauschecker, J.P., 1998. Cortical processing of complex sounds. Curr. Opin. Neurobiol. 8, 516-521.

Romanski, L.M., Tian, B., Fritz, J.B., Mishkin, M., Goldman-Rakic, P.S., Rauschecker, J.P., 1999. Dual streams of auditory afferents target multiple domains in the primate prefrontal cortex. Nat. Neurosci. 2, $1131-1136$.

Romanski, L.M., Tian, B., Fritz, J.B., Mishkin, M., Goldman-Rakic, P.S., Rauschecker, J.P., 2000. Reply to "“what', 'where' and 'how' in auditory cortex." Belin and Zatorre (2000). Nat. Neurosci. 3, 966.

Scott, S.K., Blank, C.C., Rosen, S., Wise, R.J.S., 2000. Identification of a pathways for intelligible speech in the left temporal lobe. Brain 123, $2400-2406$. 
Shapleske, S., Rossel, S.L., Woodruff, P.W.R., 1999. The planum temporale: a systematic quantitative review of its structural, functional and clinical significance. Brain. Res. Rev. 29, 26-49.

Symms, M.R., Barker, G.J., Franconi, F., Clark, C.A., 1997. Proceedings of the Annual Meeting of the ISMRM 1723. ISMRM, Vancouver.

Talairach, J., Tournoux, P., 1988. Co-Planar Stereotaxic Atlas of the Human Brain. Georg Thieme Verlag, Stuttgart.

Toga, A.W., Thompson, P.M., 2003. Mapping brain asymmetry. Nat. Rev., Neurosci. 4, 37-48.

Tuch, D.S., Reese, T.G., Wiegell, M.R., Makris, N., Belliveau, J.W., Weeden, V.J., 2002. High angular resolution diffusion imaging reveals intravoxel white matter fiber heterogeneity. Magn. Reson. Med. 48, 577-582.
Tuch, D.S., Reese, T.G., Wiegell, M.R., Wedeen, V.J., 2003. Diffusion MRI of complex neural architecture. Neuron 40, 885-895.

Wheeler-Kingshott, C.A.M., Boulby, P.A., Symms, M.R., Barker, G.J., 2002. Optimised cardiac gating for high angular-resolution wholebrain DTI on a standard scanner. Proc. Int. Soc. Magn. Reson., 1118.

Wise, R., 2003. Language systems in normal and aphasic human subjects: functional imaging studies and inferences from animal studies. Br. Med. Bull. 65, 95-119.

Wise, R., Scott, S., Blank, S., Mummery, C.J., Murphy, K., Warburton, E., 2001. Separate neural subsystems within 'Wernicke's area'. Brain 124, $83-95$. 